\title{
Luxationen und Frakturen im Lisfranc-Gelenk
}

\author{
Volker Bühren
}

\section{Zusammenfassung}

Als Lisfrancsche Gelenklinie wird die Verbindung der 5 Mittelfußknochen an die eigentliche Fußwurzel bezeichnet. Reine ligamentäre Verletzungen sind selten, meistens handelt es sich um Luxationsfrakturen mit Schaftbrüchen der Mittelfußknochen und Impressionen und knöchernen Ausrissen an der Fußwurzel. Wegen der komplexen Anatomie empfiehlt sich immer die Abklärung mit Computertomogramm. Luxationsfrakturen werden auf heutigem Stand meist operativ mittels Spickdrähten und Einzelschrauben versorgt. Bei frühzeitiger Osteosynthese, konsequentem Weichteilmanagement und korrekter Nachbehandlung ist ein gutes Funktionsergebnis zu erwarten.

\section{Einleitung}

Die Gelenklinie, die von den 5 kleinen Röhrenknochen des Mittelfußes und den 4 Knochen der distalen Fußwurzelreihe gebildet wird, ist nach Jacques Lisfranc genannt, der von 1790 bis 1847 gelebt hat und Feldchirurg in den Napoleonischen Armeen war. Lisfranc hat diese Gelenklinie für Amputationen des Vorfußes benutzt. Die Behandlung von Frakturen und Luxationen wurde von ihm nicht beschrieben.

Im Querschnitt bilden die 5 kleinen Röhrenknochen des Mittelfußes einen Halbkreis, der mit einem römischen Torbogen verglichen werden kann (Abb.1).

OP-JOURNAL 2001; 17: 56-59

(C) Georg Thieme Verlag Stuttgart · New York

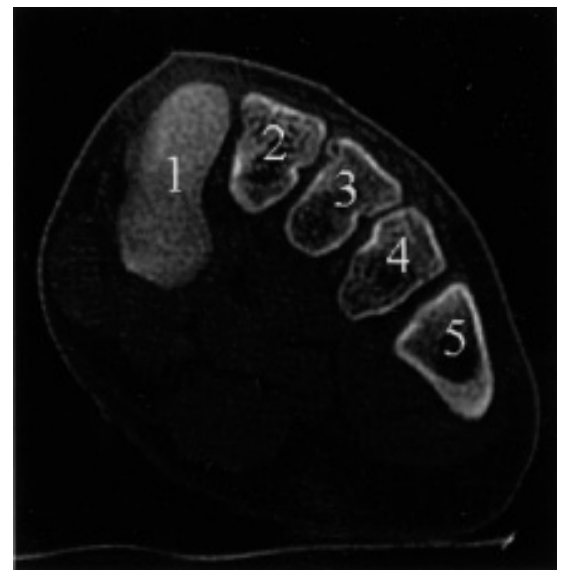

Abb. 1 CT-Schnittbild des Fußskeletts in Höhe der Basis der 5. Mittelfußknochen. Es zeigt sich die Bogenstruktur mit MFK II als Schlüsselstein.

Der II. Mittelfußknochen hat dabei als so genannter Schlussstein eine Schlüsselstellung.

Diese behält er im übrigen auch für die operative Versorgung bei. Dieser Mittelfußknochen muss immer zuerst reponiert werden, die Reposition der übrigen Knochen ist in der Folge erleichtert.

Der mediale Anteil der Gelenklinie wird vom Großzehenstrahl mit dem ersten Os cuneiforme gebildet. Den mittleren Anteil bilden der II. und III. Mittelfußknochen (MFK), die jeweils auf das gleichnamige Os cuneiforme treffen. Die laterale Artikulation wird zwischen 4. und 5. MFK und dem Os cuboideum gebildet. Sämtliche genannten Knochen sind untereinander durch sehr straffe Bandzüge verbunden, die für jedes Einzelgelenk nur geringe Bewegungsausmaße erlauben. Die eigentliche Funktion entsteht durch das Zusammenspiel aller Gelenke in der Funktionseinheit Vor- und Mittelfuß.

\section{Klassifikation}

Die erste Klassifikation, die heute noch benutzt wird, stammt von den Erstbeschreibern QUENU und KÜSS schon aus 1909 [8]. Diese haben 3 Grundluxationsrichtungen definiert (Abb. 2). Bei der homolateralen Grundform findet eine gleichförmige Luxation aller 5 Mittelfußknochen meist nach lateral statt. Bei der partiellen Luxationsform luxiert nur der I.Strahl nach medial. Bei der divergierenden Luxationsform findet sich eine Spaltung der Fußwurzelreihe, wobei der 1. oder der 2.Strahl im Verband nach medial abweichen und die übrigen Strahlen noch lateral luxieren.

Insgesamt gesehen ist die Lisfranc-Luxationsfraktur selten und macht im FußSprunggelenksbereich nur etwa $4 \%$ der Frakturen aus. In der Mehrzahl der Fälle mit $2 / 3$ liegen Hochrasanztraumen vor, z.B. Verfangen des Fußes im Pedalraum des Pkw, oder Stürze aus größerer Höhe oder schwere Quetschverletzungen [10]. Allerdings treten Lisfranc-Luxationsfrakturen auch bei Niederrasanztraumen auf, dann oft nur mit dezenter Fehlstellung und entsprechend erschwerter Diagnostik [5].

\section{Diagnostik}

Die Lisfrancsche Luxationsfraktur stellt eine der klassischen Verletzungsformen dar, die häufiger diagnostisch übersehen werden.

Dies liegt zum einen in der recht komplexen Röntgenanatomie mit oft diskreten Fehlstellungen, die missinterpretiert werden können. Zum anderen finden sich derartige Verletzungen gehäuft bei Polytraumen mit einem Anteil bis zu $50 \%$. Bei diesen Patienten stehen andere Verletzungen vital im Vordergrund, die Schwellung im Vorfußbereich fällt zunächst nicht auf und wird entsprechend dann diagnostisch nicht abgeklärt. 


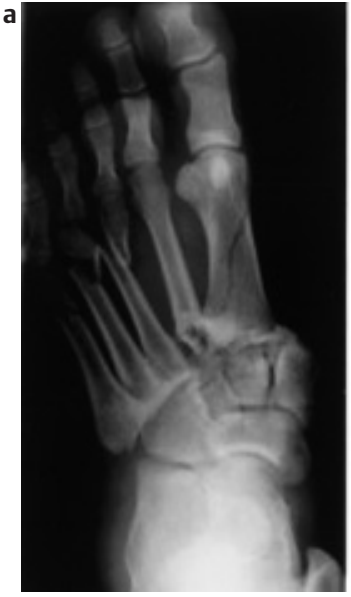

Abb. 2 Fallbeispiele für die 3 häufigsten Luxationsformen: (a) homolaterale Verrenkung aller 5 Mittelfußknochen typischerweise nach lateral, (b) partielle Verrenkung des 1. Strahls nach medial, deutlich zu erkennen die deut-

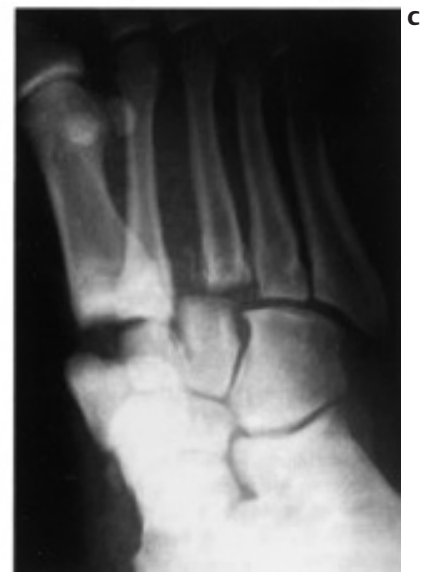

liche Lücke zwischen 1. und 2.Strahl im Vergleich zur Abb. 1 mit anatomischer Stellung, (c) divergierende Luxation mit Auseinanderweichen von 2. und 3. Strahl, wobei die äußeren Strahlen typischerweise nach lateral luxieren.

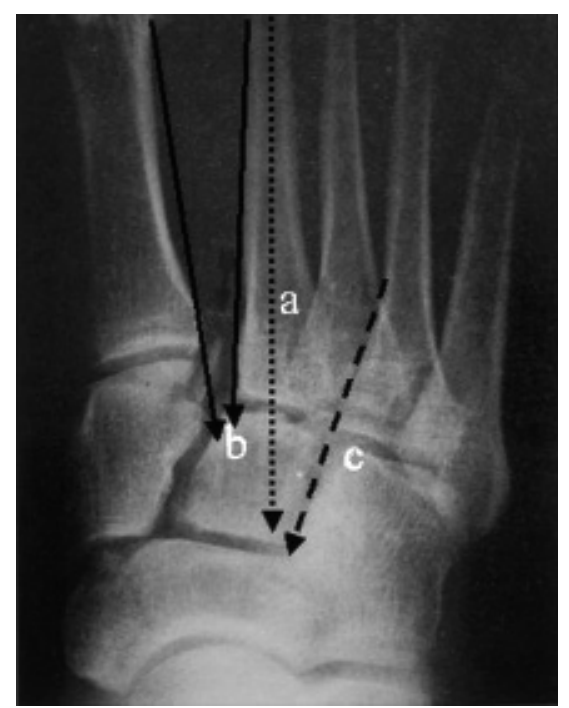

Abb. 3 Hilfslinien zur Erkennung diskreter Fehlstellungen der Lisfrancschen Gelenklinie, Erläuterungen siehe Text.

Standarddiagnostik ist die dorso-plantare Aufnahme mit 20 Grad gekippter Röhre, so dass eine regelrechte a.p.-Projektion resultiert. In der 2.Projektion erfolgt zumeist eine Schrägaufnahme des Fußes, die besseren Einblick in die Gelenke erbringen soll. Größere diagnostische Sicherheit bringt eine exakte seitliche Aufnahme des Fußes, die Verwerfungen der Gelenklinie erkennen lässt [3].

Die Orientierung kann mittels Hilfslinien erfolgen (Abb. 3). Dabei muss die zentrale Achse des 2. Mittelfußknochens exakt mit seiner Verlängerung in der Mitte der dis-
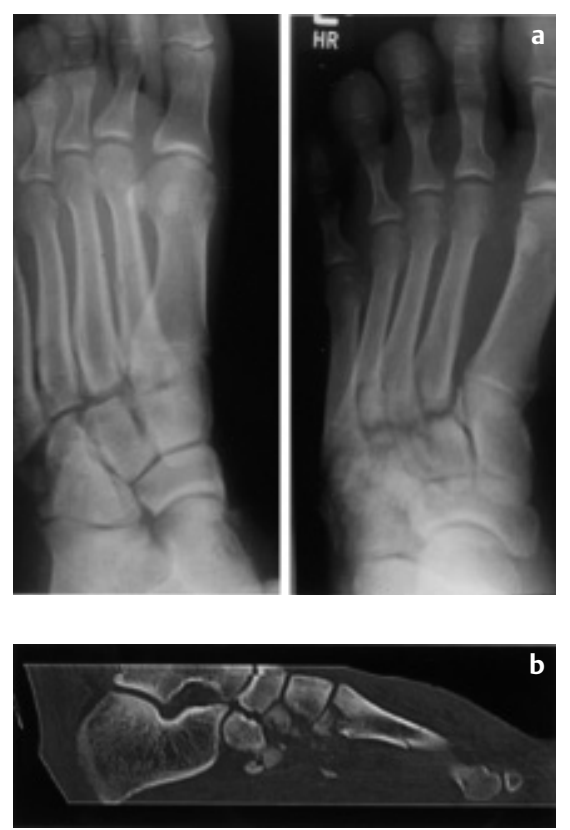

Abb.4 Im Röntgenbild a diskrete Frakturzeichen in der Lisfrancschen Gelenklinie, im CT b und c zeigt sich das Vollausmaß der Gelenk-

talen Gelenklinie des Cuneiforme II verlaufen (Abb.3a). Der Abstand zwischen 1. und 2.MFK sollte an der Basis nicht mehr als $3 \mathrm{~mm}$ betragen (Abb. $\mathbf{3 b}$ ). Einen guten Anhalt ergibt auch die Linie entlang der medialen Kortikalis des 4.MFK, die exakt in der Verlängerung der medialen Kortikalis des Cuboids enden muss (Abb. 3c).

Bei Zweifeln über das Verletzungsausmaß und zur Abklärung insbesondere vor operativen Eingriffen ist heute immer ein $\mathrm{CT}$ zu fordern.

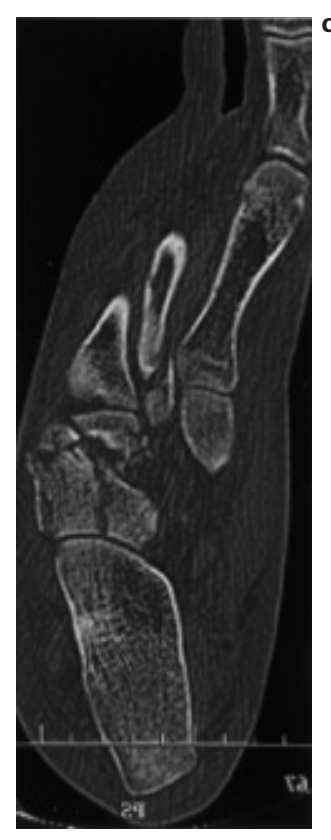

fraktur mit knöchernen Abrissen und Fissuren insbesondere in den angrenzenden Fußwurzelknochen.

Dieses gibt im Gegensatz zu den Übersichtsaufnahmen einen genauen Anhalt zum Verletzungsausmaß und insbesondere zur Stellung der Skelettanteile untereinander (Abb.4).

\section{Versorgungsprinzipien}

Bei rein ligamentären Luxationen kann eine gedeckte Reposition versucht werden, die insbesondere im akuten Zustand gut gelingen kann. Ist die Reposition stabil, kann konservativ mit Gipsruhigstellung weiterbehandelt werden. Bei Verdacht auf instabile Retention sollte im- 

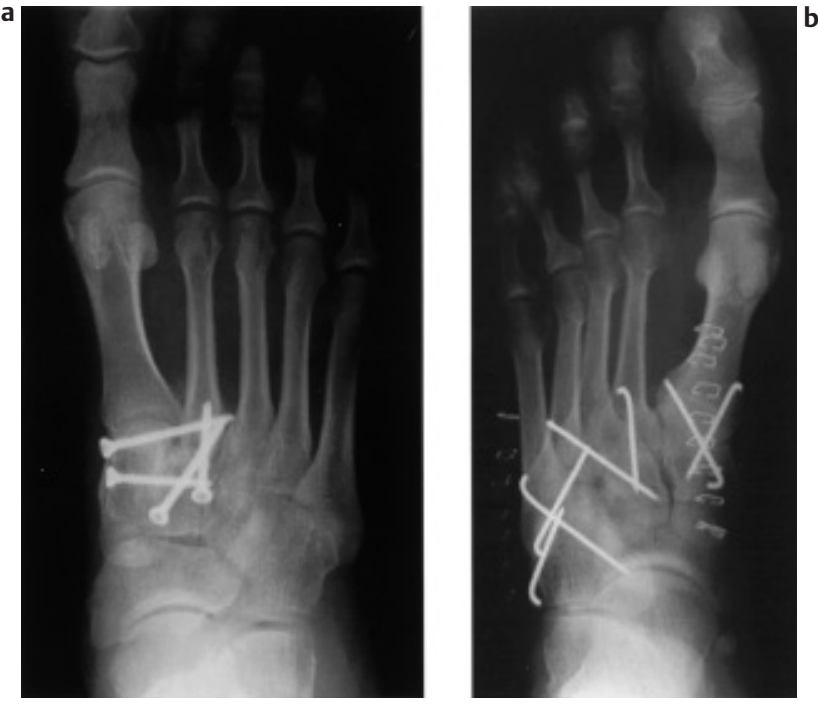

Abb.5 Fallbeispiele für die Fixierung der Gelenkluxationen: (a) Arthrodese von Cuneiforme I auf Cuneiforme II sowie des Basisgelenkes zwischen MFK II und Cuneiforme II. Zu beachten ist, dass das Basisgelenk des 1. Strahls freigelassen wurde. (b) Kirsch-DrahtOsteosynthese der gesamten Lisfrancschen Gelenklinie, wobei jedes einzelne Gelenk einzeln mit kurzen Drähten fixiert wurde. In diesem Falle Zugehen über 2 getrennte Inzisionen.
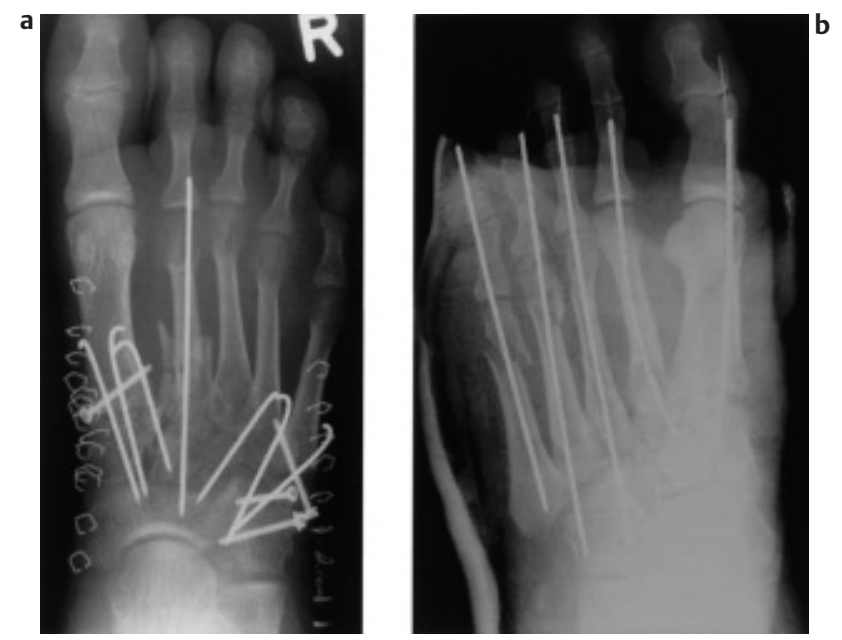

Abb. 6 Fallbeispiele für komplexe Versorgungen: (a) komplexe Luxationsfraktur der gesamten Gelenklinie mit Schaftfraktur MFK II.Schraubenosteosynthese der knöchernen Bandausrisse und Impressionen an MFK I und am Cuboid. Sämtliche Einzelgelenke sind mittels Spickdrähten transfixiert, die Schaftfraktur am II. Strahl mittels langem Spickdraht intramedullär geschient. (b) Simultanversorung einer Serienfraktur der Mittelfußknochen gleichzeitig mit Versorgung der Lisfranc-Luxation, indem sämtliche Spickdrähte gleichzeitig die Basisgelenke transfixieren.

mer eine gedeckte Spickung der anatomischen Stellung erfolgen.

In der Mehrzahl der Fälle liegen Luxationsfrakturen vor, die nach heutiger Auffassung eine operative Versorgung benötigen.
Ziel ist dabei die exakte Wiederherstellung der Gelenkform und insbesondere des schon zitierten Torbogens für die Mittelfußreihe. Die Reposition gelingt im Akutstadium deutlich einfacher, so dass heute ganz überwiegend die Frühversorgung angestrebt wird. Bei Polytraumati- sierung kann die Versorgung in einem Intervall bis zu einer Woche ohne Nachteile für die Reponierbarkeit erfolgen.

Nach mehr als 1 Woche und insbesondere bei verspäteten Fällen ist die Reponierbarkeit durch Weichteilretraktion deutlich behindert und schwieriger. Als operativer Zugang wird ein dorsaler Längsschnitt zwischen MFK II und III empfohlen, der einen guten Zugang zum Schlüsselknochen MFK II sowie zur medialen Gelenkseite erlaubt. Luxationen lateral insbesondere des 5.MFK müssen bisweilen perkutan über Hilfsschnitte versorgt werden. Alternativ können auch 2 Zugänge lateral und medial verwendet werden, wobei die Hautbrücke breit genug zu wählen ist.

Besondere Aufmerksamkeit erfordert das Weichteilmanagement. Ein Hautverschluss darf nicht erzwungen werden.

Bei hoher Weichteilspannung empfiehlt sich eine temporäre Deckung, z.B. auch als Vakuumversiegelung. Dies stellt auch gleichzeitig eine wirksame Prophylaxe für die Entstehung eines Fußcompartements dar [9].

Für die Osteosynthese werden KirschnerDrähte der Stärken 1,2 bis $2,0 \mathrm{~mm}$ benutzt. Für die Therapie der knöchernen Bandausrisse und Impaktionen an den Fußwurzelknochen hat sich die Verwendung von Kleinfragmentschrauben bewährt. Das Versorgungsprinzip besteht in einer Transfixation der Gelenke, die bei Trümmerfrakturen im Sinne einer Arthrodese belassen wird, bei Gelenkluxationen nur temporär durchgeführt wird (Abb.5). Oft müssen Schaftbrüche der Mittelfußknochen mitversorgt werden. Hierfür eignen sich insbesondere Kirschner-Drähte der Stärke $2,0 \mathrm{~mm}$, die im Sinne einer intramedullären Osteosynthese gleichzeitig die Schaftfraktur schienen und durch Weiterbohren eine temporäre Transfixierung sicher stellen (Abb. 6).

Für die Nachbehandlung hat sich eine Gipsruhigstellung für 2 Wochen bewährt, anschließend kann je nach Ausdehnung des Befundes im Vorfußentlastungsschuh aufbelastet werden. Eine Entfernung der Transfixation sollte frühestens nach 6 Wochen erfolgen. Die Vollbelastung ist bei kallöser Überbauung der Frakturen, in der Regel nach der 8.Woche, möglich. 


\section{Prognose}

Ergebnisse nach operativer Versorgung von Lisfranc-Luxationsfrakturen werden von diversen Autoren mit meist kleinen Kollektiven berichtet $[1,2,4]$. Wegen der Seltenheit der Verletzung liegen große Sammelstudien nicht vor.

Fasst man neuere Ergebnisse zusammen, zeigen sich bei über $90 \%$ der Patienten unter der Voraussetzung einer frühzeitigen operativen Versorgung gute und befriedigende Ergebnisse.

Schlechte Ergebnisse resultieren regelmäßig aus verbliebenen Fehlstellungen, die persistierende Instabilitäten bedingen. Für diese Fälle ist eine operative Revision mit Umstellungsosteotomie und Teilarthrodese angezeigt [6,7].

\section{Fazit}

Bei konsequenter Diagnostik und Therapie können Lisfranc-Frakturen mit relativ einfachen Maßnahmen zu einem günstigen Ausheilungsergebnis geführt werden. Die Problematik liegt zum einen im Übersehen der Frakturen nach banalen Traumen und beim Polytrauma. Abhilfe schaffen hier nur das „DranDenken“ sowie eine konsequente Röntgendiagnostik mit Einstellung des Fußes in 3 Ebenen und einer großzügigen $\mathrm{Ab}$ klärung mittels CT. In der Regel erfordern die vorliegenden Frakturen ein offenes Vorgehen. Ziel ist eine anatomische Reposition der Gelenklinie und eine osteosynthetische Schienung aller Begleitfrakturen. Entscheidend für einen infektfreien Verlauf ist das Weichteilmanagement, bei dem ein primärer Hautverschluss nie erzwungen werden darf.

\section{Literatur}

${ }^{1}$ Arntz CT, Veith RG, Hansen ST. Fractures and Fractur-Dislocations of the Tarsometatarsal Joint. J Bone Joint Surg 1988; 70-A: 173-181

2 Brunet JA, Wiley JJ. The late Results of Tarsometatarsal Joint Injuries. J Bone Joint Surg 1987; 69-B: 437 - 440

${ }^{3}$ Goossens M, De Stoop N. Lisfranc's FractureDislocations: Etiology, Radiology, and Results of Treatment.Clin Orthop 1993; 176: 154- 162

${ }^{4}$ Hardcastle PH, Reschauer R, Kutscha-Lissberg E, Schoffmann W. Injuries to the Tarsometatarsal Joint. J Bone Joint Surg 1982; 64 B: $349-356$

${ }^{5}$ Faciszewski T, Burks RT, Manaster BJ. Subtle Injuries of the Lisfranc Joint. J Bone Joint Surg 1990; 72-A: 1519-1522

${ }^{6}$ Komenda GA, Myerson MS, Biddinger KR. Results of Arthrodesis of the Tarsometatarsal Joint after Traumatic Injury. J Bone Joint Surg 1996; 78-A: 1665-1676

${ }^{7}$ Mann RA, Prieskorn D, Sobel M. Mid-Tarsal and Tasometatarsal Arthrodesis for Prima- ray - Degenerative Osteoarthrosis or Osteoarthrosis after Trauma. J Bone Joint Surg 1996; 78-A: 1376-1385

${ }^{8}$ Quenu E, Küss G. Etude sur les luxations de metatarse (luxations metatarso-tarsiennes) du diastasis entre le 1 et le 2 metatarsien. Rev Chir 1909; 39: 281-336, 720-791, 1093 - 1134

${ }^{9}$ Suren EG, Zwipp H. Luxationsfrakturen im Chopart- und Lisfranc-Gelenk. Unfallchirurg 1989; 92: 130-139

${ }^{10}$ Vuori JP, Aro HT. Lisfranc Joint Injuries: Trauma Mechanisms and Associated Injuries. J Trauma 1993; 35: 40-45

Prof. Dr. med. Volker Bühren Ärztlicher Direktor

BG-Unfallklinik Murnau Professor-Küntscher-Str. 8 82418 Murnau 